\title{
A Novel High Intensity Focused Ultrasound Robotic System for Breast Cancer Treatment
}

\author{
Taizan Yonetsuji ${ }^{1}$, Takehiro Ando ${ }^{1}$, Junchen Wang ${ }^{1}$, Keisuke Fujiwara ${ }^{2}$, \\ Kazunori Itani ${ }^{2}$, Takashi Azuma ${ }^{1}$, Kiyoshi Yoshinaka ${ }^{3}$, Akira Sasaki ${ }^{1}$, Shu Takagi ${ }^{1}$, \\ Etsuko Kobayashi ${ }^{1}$, Hongen Liao ${ }^{1}$, Yoichiro Matsumoto ${ }^{1}$, and Ichiro Sakuma ${ }^{1}$ \\ ${ }^{1}$ Graduate School of Engineering, The University of Tokyo, Japan \\ ${ }^{2}$ Hitachi Aloka Medical, Ltd., Japan \\ ${ }^{3}$ Advanced Industrial Science and Technology, Japan
}

\begin{abstract}
High intensity focused ultrasound (HIFU) is a promising technique for cancer treatment owing to its minimal invasiveness and safety. However, skin burn, long treatment time and incomplete ablation are main shortcomings of this method. This paper presents a novel HIFU robotic system for breast cancer treatment. The robot has 4 rotational degrees of freedom with the workspace located in a water tank for HIFU beam imaging and ablation treatment. The HIFU transducer combined with a diagnostic 2D linear ultrasound probe is mounted on the robot end-effector, which is rotated around the HIFU focus when ablating the tumor. HIFU beams are visualized by the $2 \mathrm{D}$ probe using beam imaging. Skin burn can be prevented or alleviated by avoiding long time insonification towards the same skin area. The time cost could be significantly reduced, as there is no need to interrupt the ablation procedure for cooling the skin. In addition, our proposed robot control strategies can avoid incomplete ablation. Experiments were carried out and the results showed the effectiveness of our proposed system.
\end{abstract}

\section{Introduction}

Breast cancer is a type of cancer originating from breast tissue and most likely occurs in women. It accounts for $22.9 \%$ of all cancers in women worldwide in 2008 which has been thought as the biggest health threat to women among all cancers. The treatments for breast cancer include surgery, medications, radiation and/or immunotherapy, among which, surgery (e.g. mastectomy, quadrantectomy) is the most reliable treatment to increase the cure rate and prevent recurrence. However, the removal or partial removal of breast significantly affects the QOL (Quality of Life) in patients. Therefore, breast-conserving surgery has been drawing great attentions in the biomedical engineering community. As a type of breast-conserving surgery, high intensity focused ultrasound (HIFU) has become available for breast cancer treatment. HIFU can focus ultrasound at its focal point and direct acoustic energy into the focus. In HIFU treatment, the focused acoustic energy in human body is absorbed by tissue and is converted into heat so as to ablate tumors. Since only the focus area at a depth inside the body is heated while leaving the superficial skin intact, HIFU is considered as 
a non-invasive or minimally invasive medical procedure for precise tumor ablation such as breast cancer, liver cancer, prostate cancer, etc.

Apart from the accuracy in position control of the HIFU focus, another two concerns about HIFU treatment are skin burn and ablation monitoring [1]. Although most of the acoustic energy is directed to the focus during the insonification, there is still a risk of skin burn if long-time high acoustic energy is delivered for effective ablation. In order to avoid skin burn, the ablation process has to be interrupted periodically waiting for heat dissipation of the skin, which increases the treatment time. In addition, HIFU itself cannot provide the image of the ablation area and it is usually used with other imaging modalities for identifying the target and monitoring the ablation process. Magnetic Resonance-guided HIFU (MRgHIFU) [2] and ultrasound-guided HIFU (USgHIFU) [3] are two most common types of HIFU treatment systems. The former uses intra-operative MR images and the latter uses ultrasound images to monitor the ablation process. Although intra-operative temperature monitoring (updated every several seconds) is possible by using MR thermometry, the limitations of MRgHIFU still include the oversize of the MR scanner, the complicated electronic control for HIFU focusing, and the high manufacture and treatment cost. In contrast, USgHIFU has promising potentials in terms of its "real" real-time imaging, compactness and low cost. Some robotic systems for HIFU treatment were also proposed to enable automatic and precise HIFU transducer positioning [4, 5]. However, these studies only focus on the transducer positioning by robotic systems. The robots are only used as automatic transducer holders. The mentioned concerns on skin burn and long treatment time remain unsolved.

In this paper, we propose a novel HIFU robotic system for non-invasive breast cancer treatment with effective ablation and without skin burn. A HIFU transducer for ablation and a diagnostic linear 2D ultrasound probe for real-time imaging (imaging of both the HIFU beams and the target tumor) are combined together and attached to the robot end-effector. The large movement of the HIFU focus is executed by the robot arm and the small adjustment by electronic control, which only needs 56 piezoelectric elements to constitute the HIFU transducer. Skin burn is prevented by varying insonification region of the breast skin while keeping the focus (ablation area) beneath the skin unchanged or moving under control according to our proposed ablation strategies. The robotic system is designed to work under water for ultrasound compatibility.

\section{System Design}

\subsection{System Overview}

In HIFU treatment, the gap between the HIFU transducer and the human body must be filled with water or gels for ultrasound propagation. Although a water bag may be used for this purpose, the bag will touch against the breast and cause breast deformation. In addition, the HIFU transducer also has to come in contact with the bag causing our concept of rotary insonification to be unfeasible. Thus the workspace of our robot is designed under water. Because the skin burn is caused by heat accumulation 
on the skin surface covered by the insonification, it is possible to reduce the heat accumulation by moving the insonification region while keeping the focus unchanged. Based on this idea, we designed a novel articulate robot that can perform rotary insonification under water. As illustrated in Fig. 1, our system consists of an operation table on which a patient lies in the prone position, a robotic arm performing rotary insonification, a HIFU transducer that is integrated with a diagnostic ultrasound probe, a diagnostic ultrasound machine to which the probe is connected, and some control units. The spatial relationship between the diagnostic probe and the HIFU probe is calibrated so that the beams emitted by the HIFU probe can be imaged by the diagnostic probe (beam imaging), which enables visualizing both the focus (intersection of HIFU beams) and the tumor on the ultrasound machine. The robotic arm enables precise focus positioning. Furthermore, our novel ablation strategy can reduce the heat accumulation and treatment time while performing efficient ablation.

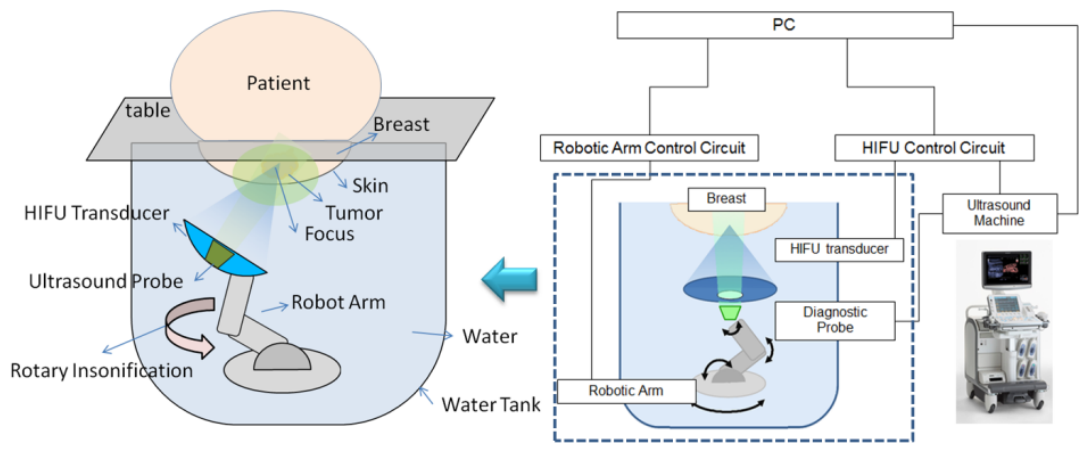

Fig. 1. System overview

\subsection{Integrated HIFU Transducer}

The function of a HIFU transducer is to focus HIFU beams at its focal point so that most acoustic energy is delivered to the focus to ablate tumors. By moving the focus, a volume thus can be ablated. However, the focus is below the skin and is invisible to surgeons, which makes it difficult to locate the HIFU focus to the target tumor. Unlike the diagnostic ultrasound, the HIFU transducer has no imaging functionality. First, the visualization of the HIFU beams should be solved.

We developed an integrated HIFU transducer that enables accurate and registration-free focus localization. The integrated HIFU transducer consists of a multichannel piezoelectric element array for HIFU beam generation, a diagnostic ultrasound probe for real-time imaging, and peripheral circuit. The transducer has a diameter of $100 \mathrm{~mm}$ with a focal length of $100 \mathrm{~mm}$. The irradiation frequency is $2 \mathrm{MHz}$. The diagnostic ultrasound probe is fixed at the center of the transducer as shown in Fig. 2(a). HIFU beams are imaged using the diagnostic probe by receiving reflected acoustic waves that are emitted from the HIFU transducer. The principle and the imaged HIFU beams are shown in Fig. 2(b). More detailed information about beam imaging can be found in our previous paper [6]. By this way, the target tumor and the 
HIFU beams can be simultaneously visualized in real-time on the diagnostic ultrasound machine. Since all spatial information regarding the focus and the tumor is visible to surgeons, registration becomes unnecessary. The integrated transducer is attached to the robot end-effector and the HIFU focus is adjusted and located to the target tumor by visual feedback. Afterwards, the robot is driven to perform rotary insonification.

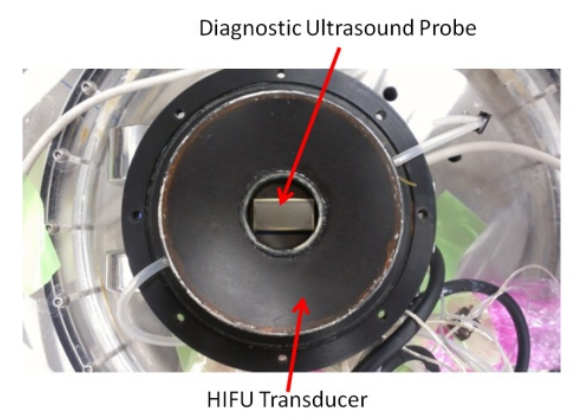

(a)

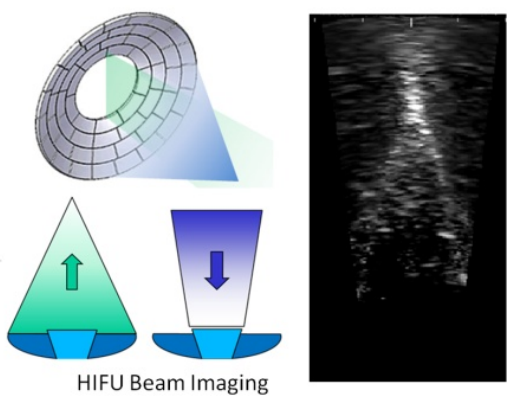

(b)

Fig. 2. Integrated transducer (a) Picture of integrated transducer (b) HIFU beam imaging

\subsection{Robot Arm}

We developed a chain-driven articulated robot with 4 degrees of freedom that works in a water tank. Fig. 3(a) shows the design drawing and the Denavit-Hartenberg (DH) model of our robot, and Fig. 3(b) shows its physical setup. It has two serial links with the same length and four joints, which are inside the water tank. The driving motors with encoders are placed under the water tank. Because the driving force of each joint is transmitted by gears and chains, which can work under water, only the motor shafts need water sealing. The diameter of the water tank is $600 \mathrm{~mm}$ and the height including motor units is $775 \mathrm{~mm}$, which can be easily moved by a cart.

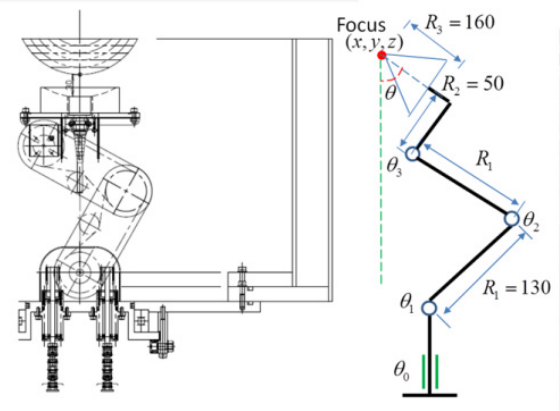

(a)

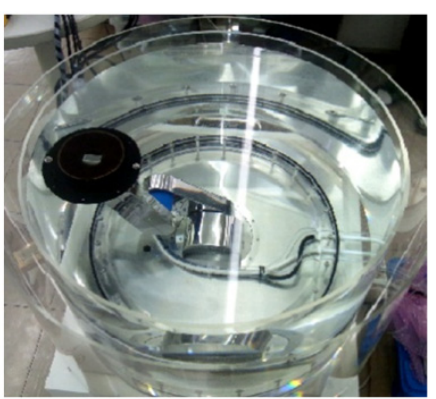

(b)

Fig. 3. Robot arm (a) Design drawing and DH model (b) Physical setup 
In order to locate the focus in the 3D space within the water tank, the robot is controlled using inverse kinematics. The rotation angle of each joint can be calculated:

$$
\left(\begin{array}{l}
\theta_{0} \\
\theta_{1} \\
\theta_{2} \\
\theta_{3}
\end{array}\right)=\left(\begin{array}{c}
\arccos \left(\frac{x}{\sqrt{x^{2}+y^{2}}}\right) \\
\arccos \left(\frac{z-\left(R_{2} \sin \theta_{3}+R_{3} \cos \theta_{3}\right)}{r}\right)+\arccos \left(\frac{r}{2 R_{1}}\right) \\
\arccos \left(\frac{z-\left(R_{2} \sin \theta_{3}+R_{3} \cos \theta_{3}\right)}{r}\right)-\arccos \left(\frac{r}{2 R_{1}}\right) \\
\theta
\end{array}\right)
$$

Where $(x, y, z, \theta)$ represents the target pose of the transducer (see Fig. 3(a)), $\left(\theta_{0}, \theta_{1}, \theta_{2}, \theta_{3}\right)$ are the rotation angles of each joint, $r=x^{2}+y^{2}+z^{2}+R_{2}{ }^{2}+$ $R_{3}{ }^{2}+2\left(R_{2} \sqrt{x^{2}+y^{2}}-R_{3} z\right) \sin \theta_{3}-2\left(R_{3} \sqrt{x^{2}+y^{2}}+R_{2} z\right) \cos \theta_{3}, R_{1} \sim R_{3}$ are link lengths with $R_{1}=130 \mathrm{~mm}, R_{2}=50 \mathrm{~mm}$, and $R_{3}=160 \mathrm{~mm}$.

\subsection{Rotary Insonification Ablation}

In this study, we propose a rotary insonification procedure that could prevent the skin burn without losing ablation efficiency. Since the skin burn is caused by the lasting insonification towards the same skin area, it is possible to reduce the heat accumulation by varying the insonification region of acoustic energy during the ablation. There are two modes of rotary insonification as illustrated in Fig. 4: 1. Rotate the transducer around the vertical axis passing through the focus (Fig. 4(a)). 2. Rotate the transducer around the vertical axis deviated from the focus (Fig. 4(b)). In Mode 1, the insonification region of the superficial skin varies over time while keeping the focus unchanged; in Mode 2, the focus also moves (rotation) in order to cover a large ablation volume. Both of the two modes can alleviate the heat accumulation by avoiding the stationary lasting insonification towards the same skin area.
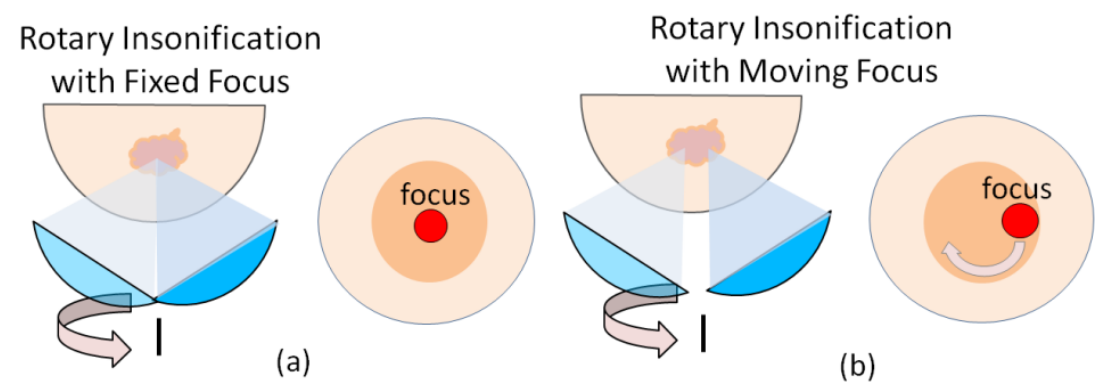

Fig. 4. Rotary insonification (a) With fixed focus (b) With moving focus

\section{Experiments}

\subsection{Evaluation of Robot Accuracy}

The rotation accuracy of each robot joint was evaluated. An optical tracking system (Polaris, NDI) was used to produce ground truth. A 6DOF optical marker was 
attached to the end-effector of the robot. Rotation accuracy was evaluated by comparing the actual rotation angles reported by Polaris with the input values. The evaluation was performed for ten times every 10 degrees for each joint and the angle errors were calculated. Table 1 summarizes the statistic results of rotation angle errors. $\Delta \theta_{i}$ represents the rotation angle error of joint $i$. (+) means forward rotation; (-) means backward rotation. The errors for forward and backward rotation are different owing to the backlash of the chains. STD stands for standard deviation.

Table 1. Rotation angle error (degree)

\begin{tabular}{c|ccccccc}
\hline & $\Delta \theta_{0}$ & $\Delta \theta_{1}(+)$ & $\Delta \theta_{1}(-)$ & $\Delta \theta_{2}(+)$ & $\Delta \theta_{2}(-)$ & $\Delta \theta_{3}(+)$ & $\Delta \theta_{3}(-)$ \\
\hline Average & 0.53 & 0.22 & 0.97 & 0.56 & 1.32 & 0.12 & 1.04 \\
STD & 0.098 & 0.29 & 1.03 & 0.71 & 0.95 & 0.16 & 1.01 \\
Maximum & 0.69 & 0.67 & 2.38 & 1.60 & 2.79 & 0.31 & 2.75 \\
\hline
\end{tabular}

\subsection{Evaluation of Skin Burn}

In order to evaluate the extent of the skin burn, temperature of the skin surface was estimated. As demonstrated in Fig. 5(a), a thermosensitive liquid crystal (TLC) sheet was placed on an acoustic absorbent that simulates the breast skin. Since the TLC sheet can sense temperature between $50 \sim 60^{\circ} \mathrm{C}$ with accuracy $1^{\circ} \mathrm{C}$ by color change, the temperature can thus be measured by a camera. The focus was adjusted at the distance of $20 \mathrm{~mm}$ under the TLC sheet. Ablation was performed during the same interval using both our rotary insonification method and a stationary insonification method. Pictures were taken using the camera as experimental results to compare the temperature distribution indicated by colors.

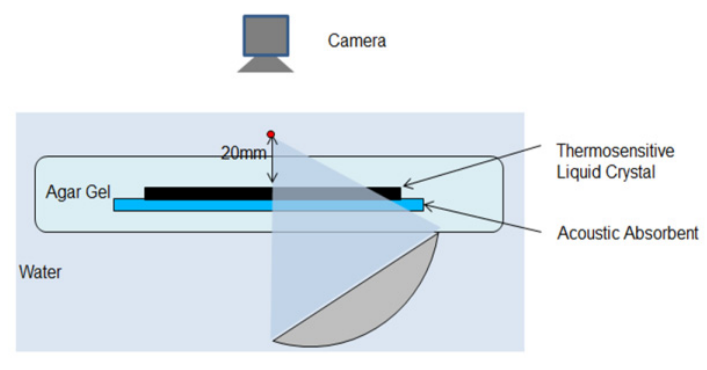

(a)

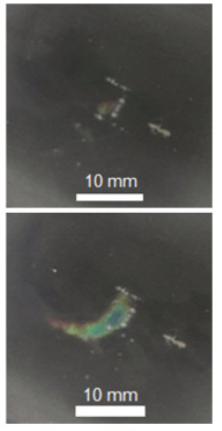

(b)

Fig. 5. Skin burn evaluation (a) Experiment design (b) Experimental results

Temperate results are shown in Fig. 5(b). The top is the result of our proposed ablation method and the bottom is the result of traditional one. It is obvious that our method successfully suppressed the heat accumulation and led to low skin temperature. The stationary insonification method heated the TLC up to over $60^{\circ} \mathrm{C}$ indicated 
by the blue region. On the other hand, although very small region became over $50^{\circ} \mathrm{C}$, our method succeeded in reducing the high temperature region.

\subsection{Evaluation of Ablation}

Ablation performance was evaluated using a breast phantom consisting of chicken breast meat and ager gel (Fig. 6). The phantom was made by firstly dissolving graphite and ager powder into deaerated water with the concentration of $1.5 \%$ and $4 \%$ respectively. Subsequently, the solution was put into a hemispherical mold with a diameter of $150 \mathrm{~mm}$ and cooled until it was solidified. Lastly, the chicken breast meat was added into the gel and was covered by pouring the solution once again onto the meat. The final breast phantom has similar shape and physical property with a real one. Here the chicken meat serves as the ablation target. The addition of graphite is to simulate the tissue scattering properties of the ultrasound.

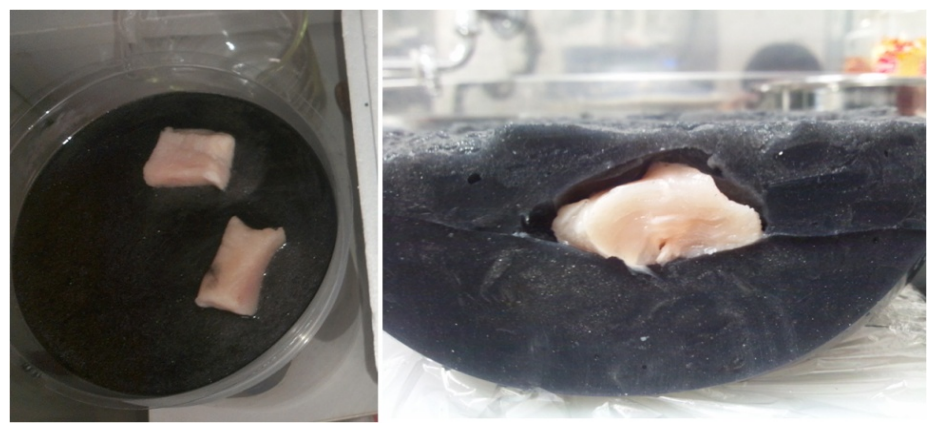

Fig. 6. Breast phantom

A single spot was ablated using rotary insonification Mode 1 described previously. Joint 0 was rotated 360 degree with the speed of 9 degree/sec while emitting HIFU beams of $4 \mathrm{~W} / \mathrm{ch}$. For comparison, the ablation was also performed under the same condition but without rotating the transducer. Next, a larger region was ablated continuously using rotary insonification Mode 2, where the focus was moved in a circle while emitting HIFU beams of $4 \mathrm{~W} / \mathrm{ch}$. Joint 0 was rotated 360 degree with the speed of 3 degree/sec, which means that the overall treatment time was 120s. The ablation using the traditional method was also performed, where seven spots were ablated separately in order to cover the entire ablation volume with 40s lasting insonification and waiting 80 s for cooling on each spot. The overall treatment time was 840 s, which was significantly longer than the proposed method.

Fig. 7(a) shows the results of the single spot ablation. The ablation area was about $12 \times 4 \mathrm{~mm}$ (left) with the stationary insonification, $8 \times 4 \mathrm{~mm}$ (right) with our method. Fig. 7(b) shows the results of the region ablation. The left is the ablation results of the stationary insonification on seven spots. The right is the results of our proposed method. We can see that our method enabled almost homogeneous ablation to avoid incomplete ablation. In contrast, the traditional method only ablated spots one by one to cover the entire target volume, resulting in inhomogeneous and incomplete ablation. 


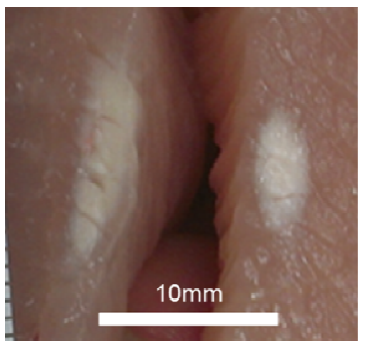

(a)

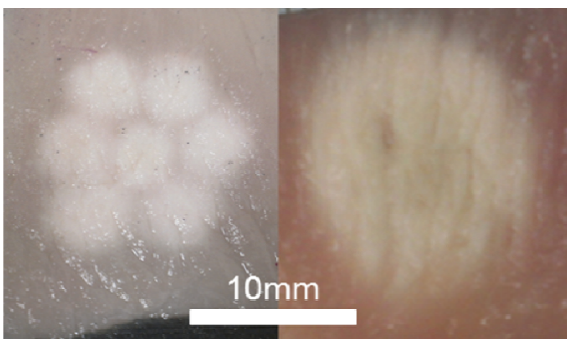

(b)

Fig. 7. Ablation evaluation (a) One spot ablation (traditional vs. proposed method) (b) Large region ablation (traditional vs. proposed method)

\section{Conclusion}

Our robot has a novel structure that works under water for ultrasound compatibility. Compared with the traditional ablation method that performs stationary insonification, our low-cost robotic system can achieve flexible rotary insonification without losing focus. The advantages of this approach include reducing the heat accumulation of the breast skin, decreasing the treatment time and enabling homogeneous region ablation. Future work includes ultrasound image-based visual servo control of the robot and HIFU beam autofocusing.

Acknowledgements. This work was supported in part by Grant for Translational Systems Biology and Medicine Initiative (TSBMI) from the Ministry of Education, Culture, Sports, Science and Technology of Japan.

\section{References}

1. Zhang, L., Zhu, H., Jin, C., et al.: High-intensity Focused Ultrasound (HIFU): Effective and Safe Therapy for Hepatocellular Carcinoma Adjacent to Major Hepatic Veins. Eur. Radiol. 19, 437-445 (2009)

2. Gianfelice, D., Khiat, A., Boulanger, Y., et al.: Feasibility of Magnetic Resonance Imaging Guided Focused Ultrasound Surgery as an Adjunct to Tamoxifen Therapy in High-risk Surgical Patients with Breast Carcinoma. J. Vasc. Interv. Radiol. 14, 1275-1282 (2003)

3. Vaezy, S., Shi, X., Martin, R., et al.: Real-time Visualization of High-intensity Focused Ultrasound Treatment Using Ultrasound Imaging. Ultrasound Med. Biol. 27(1), 33-42 (2001)

4. Kheng, N., Sing, N., Phee, L., Cheng, C.: A HIFU Robot for Transperineal Treatment of Prostate Cancer. In: Proceedings of 7th International Conference on Control, Automation, Robotics and Vision 2002, vol. 2, pp. 560-565 (2002)

5. Pather, S., Davies, B.L., Hibberd, R.D.: The Development of a Robotic System for HIFU Surgery Applied to Liver Tumours. In: Proceedings of 7th International Conference on Control, Automation, Robotics and Vision 2002, vol. 2, pp. 572-577 (2002)

6. Yonetsuji, T., Fujiwara, K., Itani, K., et al.: HIFU Beam Imaging Based on Scattering Signals from Focal Area. In: Proceedings of CIRP Conference on BioManufacturing 2013, pp. 282-285 (2013) 